\title{
Ferromagnetic ground state of an orbital degenerate electronic model for transition-metal oxides: Exact solution and physical mechanism
}

\author{
Shun-Qing Shen and Z. D. Wang \\ Department of Physics, The University of Hong Kong, Pokfulam Road, Hong Kong, People's Republic of China
}

(Received 16 July 1998)

\begin{abstract}
We present an exact ground-state solution of a one-dimensional electronic model for transition-metal oxides in the strong-coupling limit. The model contains doubly degenerated orbit for itinerant electrons and the Hund coupling between the itinerant electrons and localized spins. The ground state is proven to be a full ferromagnet for any density of electrons. Our model provides a rigorous example for metallic ferromagnetism in narrow-band systems. The physical mechanism for ferromagnetism and its relevance to high-dimensional systems, like $R_{1-x} X_{x} \mathrm{MnO}_{3}$, are discussed. Due to the orbital degeneracy of itinerant electrons, the superexchange coupling can be ferromagnetic rather than antiferromagnetic in the half-filled one-band case. [S0163-1829(99)11001-4]
\end{abstract}

The origin of ferromagnetism in narrow-band systems is a long standing problem and has recently attracted renewed attention. Essentially speaking, there are three basic routes to ferromagnetism: (1) it is formulated in the Hubbard model for an intermediate short-range Coulomb interaction with a pronounced peak in the density of state near the band edge, ${ }^{1}$ (2) the Hund's rule coupling in the presence of the orbital degeneracy of itinerant electrons leads to ferromagnetism; ${ }^{2}$ and (3) the motion of electrons in the localized spin background forces the spins to align parallelled. ${ }^{3}$ Significant progress has been made in our understanding of ferromagnetism in last several years. Recent reviews on this subject are seen in Refs. 4-6 and references therein. On the other hand, ferromagnetism in $R_{1-x} X_{x} \mathrm{MnO}_{3}$ and related ordering states have stimulated extensive interests due to the phenomenon of the colossal magnetoresistance. The physical origins still lack full understanding. Electronic models for this family of materials contain all the three factors which favor ferromagnetism, and are ideal candidates to test all physical mechanisms for ferromagnetism. Various techniques are applied to investigate magnetism in these models. ${ }^{7}$ Due to the complexity of the systems quite a few rigorous solutions or rigorous results are obtained from the models. Usually rigorous results are very helpful to shape the physics of theoretical models, especially in such a strong correlated electron system.

In this paper, we shall present an exact solution of the ground state for a one-dimensional electronic model for transition-metal oxides in the case of strong coupling. The ground states are proven to be ferromagnetic by means of the Perron-Frobenius theorem. The physical mechanism and its relevance to three-dimensional cases are discussed. Due to the orbital degeneracy of itinerant electrons, the superexchange coupling can be ferromagnetic rather than antiferromagnetic in the half-filled one-band case.

An electronic model Hamiltonian for the transition-metal oxides is defined on a discrete lattice $\wedge$ with $N_{\wedge}$ site and is written as

$$
H=H_{t}+H_{u}+H_{j}+H_{h},
$$

where

$$
\begin{gathered}
H_{t}=\sum_{i j, \gamma, \gamma^{\prime}, \sigma} t_{i j}^{\gamma \gamma^{\prime}} c_{i, \gamma, \sigma}^{\dagger} c_{j, \gamma^{\prime}, \sigma}, \\
H_{u}=\sum_{i, \gamma, \gamma^{\prime}, \sigma, \sigma^{\prime}}\left(1-\delta_{\gamma, \gamma^{\prime}} \delta_{\sigma, \sigma^{\prime}}\right) U_{\gamma \gamma^{\prime}} n_{i, \gamma, \sigma^{\prime}} n_{i, \gamma^{\prime}, \sigma^{\prime}}, \\
H_{j}=-\sum_{i, \gamma \neq \gamma^{\prime}, \sigma, \sigma^{\prime}} J_{\gamma \gamma^{\prime}}\left(c_{i, \gamma, \sigma^{\dagger}}^{\dagger} c_{i, \gamma, \sigma^{\prime}} c_{i, \gamma^{\prime}, \sigma^{\prime}}^{\dagger} c_{j, \gamma^{\prime}, \sigma}\right. \\
\left.+c_{i, \gamma, \sigma}^{\dagger} c_{i, \gamma^{\prime}, \sigma^{\prime}} c_{i, \gamma, \sigma^{\prime}}^{\dagger} c_{i, \gamma^{\prime}, \sigma^{\prime}}\right), \\
H_{h}=-\sum_{i, \gamma} J_{H} \mathbf{S}_{i} \cdot \mathbf{S}_{i, \gamma} .
\end{gathered}
$$

$c_{i, \gamma, \sigma}^{\dagger}$ and $c_{i, \gamma, \sigma}$ are creation and annihilation operators for $e_{g}$ electron with $\operatorname{spin} \sigma(= \pm 1)$ on orbital $\gamma(= \pm 1)$ at site $i$, respectively. $\mathbf{S}_{i}$ is the maximal total spin of three $t_{2 g}$ electrons $(S=3 / 2)$, and $\mathbf{S}_{i, \gamma}=\Sigma_{\sigma \sigma^{\prime}} \hat{\sigma}_{\sigma \sigma^{\prime}} c_{i, \gamma, \sigma^{\prime}}^{\dagger} c_{i, \gamma, \sigma^{\prime}} / 2$ is a spin operator for an $e_{g}$ electron and $\hat{\sigma}$ are the Pauli matrices. $H_{t}$ describes the process of electrons hopping between nearestneighbor sites. $H_{u}$ is of the on-site Coulomb interaction. $H_{j}$ is the Hund exchange coupling between itinerant electrons on different orbitals at the same site and $J_{\gamma \gamma^{\prime}}$ is always positive. $H_{h}$ is the Hund coupling between localized spins and itinerant electrons at the same sites. In this model the total spin operator

$$
\mathbf{S}_{\mathrm{tot}}=\sum_{i \in \Lambda} \mathbf{S}_{i}+\sum_{i, \gamma} \mathbf{S}_{i, \gamma}
$$

commutes with the Hamiltonian, $\left[\mathbf{S}_{\mathrm{tot}}, H\right]=0$. Hence the total spin and its $z$ component are good quantum numbers. The maximal total spin is $S_{\max }=N_{\wedge} S+N_{e} / 2\left(N_{e}\right.$ is the number of electrons and we just consider $N_{e}<2 N_{\wedge}$ ). We call the state with $S_{\max }$ a ferromagnet.

This model has been investigated by many authors. ${ }^{7}$ In order to establish some rigorous results for this model we first consider a one-dimensional open chain and focus our 
attention on the case that (i) $t_{i j}^{\gamma \gamma^{\prime}}=-t \delta_{\gamma, \gamma^{\prime}} \delta_{i, j \pm 1}$ and $t>0$; (ii) $U_{\gamma \gamma^{\prime}}=U=+\infty$ if $\gamma=\gamma^{\prime}$; (iii) $U_{\gamma \gamma^{\prime}}=U^{\prime}$ if $\gamma \neq \gamma^{\prime}$; (iv) $J_{\gamma \gamma^{\prime}}=J>0$ for $\gamma \neq \gamma^{\prime}$; (v) $J_{H}>0$. It is worth stressing that the condition (ii) excludes the double occupancy of electrons on the same orbital at the same site, which is essential to solve the one-dimensional model exactly.

To solve the model rigorously, we first investigate the ground-state properties by means of the Perron-Frobenius theorem. ${ }^{8}$ The ground state is proven to be nondegenerate and possesses the maximal total spin. In this case we can write down the ground-state wave function explicitly by utilizing the Bethe ansatz. The solution is valid for any density of electrons. The Perron-Frobenius theorem (for a real symmetric matrix) states:

Let $M=\left\{m_{i j}\right\}$ be a real, symmetric, and square matrix. If the matrix $M$ satisfies the conditions: (i) all its off-diagonal matrix elements are nonpositive, $m_{i j} \leqslant 0$; (ii) any $i$ and $j$ are connected by the matrix, i.e., we can always find an integer $n$ such that $\left(M^{n}\right)_{i j} \neq 0$, then the lowest eigenvalue is nondegenerate and all elements of the corresponding eigenvector can be taken to be positive.

This theorem was successfully applied to prove the existence of ferromagnetism in the quantum double-exchange model, ${ }^{9}$ the one-band Hubbard model, ${ }^{10}$ and the orbitally degenerate Hubbard model. ${ }^{11}$ In order to apply this theorem to the present model [Eq. (1)], we have to choose a suitable basis set to write the Hamiltonian in the form of a real, symmetric and square matrix which obeys the two conditions. Consider the system contains $N_{e}$ electrons. As the $z$ component of the total spin $S_{\text {tot }}^{z}$ is a good quantum number, the Hilbert space can be decomposed by $S_{\text {tot }}^{z}$. Assume $N_{1}$ electrons on $\gamma=-1$ orbit with spin $\sigma_{1} \leqslant \sigma_{2} \leqslant \ldots \leqslant \sigma_{N_{1}}$ are located at sites $x_{1}<x_{2}<\ldots<x_{N_{1}}$, and $N_{2}$ electrons on $\gamma$ $=1$ orbit with spin $\sigma_{N_{1}+1} \leqslant \sigma_{N_{1}+2} \leqslant \ldots \leqslant \sigma_{N_{1}+N_{2}}$ are located at sites $x_{N_{1}+1}<x_{N_{1}+2}<\ldots<x_{N_{e}} \cdot x_{m}=1, \ldots, N_{\wedge}$ and $m_{i}=0, \ldots, 2 S$. As $N_{1}-N_{2}$ is also a good quantum number, thus we choose specific $S_{\text {tot }}^{z}, N_{1}$ and $N_{2}$. (We shall show the lowest energy state is located at $N_{1}=N_{2}$ if $N_{e}$ is even, or $N_{1}=N_{2} \pm 1$ if $N_{e}$ is odd.) Denote $\alpha$ to be one of the configurations of electrons and localized spins,

$$
\begin{aligned}
|\alpha\rangle= & c_{x_{1},-1, \sigma_{1}}^{\dagger} \ldots c_{x_{N_{1}},-1, \sigma_{N_{1}}}^{\dagger} \\
& \times c_{x_{N_{1}+1}, 1, \sigma_{N_{1}+1}}^{\dagger} \ldots c_{x_{N_{e}}, 1, \sigma_{N_{e}}}^{\dagger} \\
& \times\left(\mathbf{S}_{1}^{+}\right)^{m_{1}}\left(\mathbf{S}_{2}^{+}\right)^{m_{2}} \ldots\left(\mathbf{S}_{N_{\wedge}}^{+}\right)^{m_{N_{\wedge}}}|0\rangle,
\end{aligned}
$$

where the state $|0\rangle$ has the properties:

(1) $c_{x_{m}, \gamma_{m}, \sigma_{m}}|0\rangle=0$ for any $m=1, \ldots, N_{e}$;

(2) $\mathbf{S}_{i}^{-}|0\rangle=0$ for any $i=1, \ldots, N_{\wedge}$.

As we just construct a basis within the subspace $S_{\text {tot }}^{z}$, it is required that

$$
S_{\text {tot }}^{z}=\sum_{m=1}^{N_{e}} \frac{\sigma_{m}}{2}+\sum_{i=1}^{N_{\wedge}}\left(m_{i}-S\right) .
$$

A complete set of bases consists of all possible configurations of $\left\{\left\{x_{m}\right\},\left\{\gamma_{m}\right\},\left\{\sigma_{m}\right\},\left\{m_{i}\right\}\right\}$ with the condition (2). $\alpha$ in $|\alpha\rangle$ represents one of the possible configurations.
On this basis we come to show that the Hamiltonian satisfies the condition of non-positivity and connectivity.

(1) Nonpositive off-diagonal elements: On the basis we choose, the Hamiltonian can be expressed in the form of square matrix. All nonzero off-diagonal elements of the matrix $\left\langle\alpha|H| \alpha^{\prime}\right\rangle$ are: (a) $-t$ if $x_{n}=x_{n}^{\prime} \pm 1$ and all other indices are the same; (b) $-J$ if $\sigma_{n}= \pm \sigma_{m}^{\prime}, x_{n}=x_{m}^{\prime}, \gamma_{n}=-\gamma_{m}^{\prime}$ and all other indices are the same; (c) $-J_{H}$ if $m_{i}+\sigma_{n}=m_{i}^{\prime}$ $+\sigma_{m}^{\prime}, \gamma_{n}=\gamma_{m}^{\prime}, x_{n}=x_{m}^{\prime}=i$ and all other indices are the same. All the nonzero elements are negative if $t, J, J_{H}>0$. Therefore the Hamiltonian matrix satisfies the first condition of the Perron-Frobenius theorem.

(2) Connectivity: all bases are connected through $H$ : (a) the hopping terms connect all lattice sites within the orbit $\gamma$; (b) the Hund coupling $J$ connects the two orbits at the same site; (c) the Hund coupling $J_{H}$ connects the orbits of itinerant electrons and the localized spins. Combination of (a), (b), and (c) shows all bases are connected by $H$.

On this basis, the lowest energy state with $S_{\text {tot }}^{z}$ is expressed in the form

$$
|\Psi\rangle=\sum_{\alpha} f\left(\left\{x_{m}\right\},\left\{\gamma_{m}\right\},\left\{\sigma_{m}\right\},\left\{m_{i}\right\}\right)|\alpha\rangle .
$$

According to the Perron-Frobenius theorem, we conclude that the lowest energy state with $S_{\text {tot }}^{z}$ is nondegenerate and all coefficients $f$ can be chosen to be positive, $f>0$.

Since the lowest energy state with $S_{\text {tot }}^{z}\{\mathrm{Eq}$. (3) $\}$ is nondegenerate and the total spin is a good quantum number, therefore the state must be an eigenstate of the total spin

$$
\left(\mathbf{S}_{\text {tot }}\right)^{2}|\Psi\rangle=S_{\text {tot }}\left(S_{\text {tot }}+1\right)|\Psi\rangle \text {. }
$$

To determine $S_{\text {tot }}$, we first construct an eigenstate with the maximal total spin $S_{\max }=N_{\wedge} S+N_{e} / 2$,

$$
\begin{aligned}
\mid \Phi\left(S_{\mathrm{tot}}^{z}=\right. & \left.\left.-N_{\wedge} S-N_{e} / 2\right)\right\rangle \\
= & \sum_{\left\{x_{m}\right\}} c_{x_{1},-1,-1}^{\dagger} \ldots c_{x_{N_{1}},-1,-1}^{\dagger} \\
& \times c_{x_{N_{1}+1}, 1,-1}^{\dagger} \ldots c_{x_{N_{e}, 1,-1}}^{\dagger}|0\rangle .
\end{aligned}
$$

The other $2 S_{\max }$ eigenstates with total spin $S_{\max }$ and different $z$ components are expressed by utilizing spin SU(2) symmetry

$$
\left|\Phi\left(S_{\text {tot }}^{z}\right)\right\rangle=\left(\mathbf{S}_{\mathrm{tot}}^{+}\right)^{M}\left|\Phi\left(S_{\mathrm{tot}}^{z}=-N_{\wedge} S-N_{e} / 2\right)\right\rangle=\sum_{\alpha}|\alpha\rangle,
$$

where $M=S_{\text {tot }}^{z}+N_{\wedge} S+N_{e} / 2$ and the summation runs over all possible configurations in the subspace of $S_{\text {tot }}^{z}$. The main feature of the state is that all coefficients on the basis in the subspace $S_{\text {tot }}^{z}$ are equal. This state is not orthogonal to the lowest energy state $|\Psi\rangle$ if they have the same $z$ component of total spin,

$$
\langle\Psi \mid \Phi\rangle \neq 0,
$$

since all coefficients in $|\Psi\rangle$ are positive. As both states are eigenstates of the total spin $\mathbf{S}_{\text {tot }}$, we have

$$
\left\langle\Psi\left|\left(\mathbf{S}_{\mathrm{tot}}\right)^{2}\right| \Phi\right\rangle=S_{\mathrm{tot}}\left(S_{\mathrm{tot}}+1\right)\langle\Psi \mid \Phi\rangle=S_{\max }\left(S_{\max }+1\right)\langle\Psi \mid \Phi\rangle .
$$


Hence we conclude that $S_{\text {tot }}=S_{\max }$, i.e., the lowest energy state possesses the maximal total spin. In other words, the state is fully ferromagnetic.

The lowest energy state with $S_{\text {tot }}^{z}$ is fully ferromagnetic. According to spin SU(2) symmetry of the model, the ground state is $\left(2 S_{\max }+1\right)$-fold degenerate. This property makes it possible to write down the ground- state wave function explicitly. The coefficients in the ground state are independent of spin indices, i.e.,

$$
f\left(\left\{x_{m}\right\},\left\{\gamma_{m}\right\},\left\{\sigma_{m}\right\},\left\{m_{i}\right\}\right)=g\left(\left\{x_{m}\right\},\left\{\gamma_{m}\right\}\right) .
$$

Hence the Schrödinger equation, $H|\Psi\rangle=E_{g}|\Psi\rangle$, is reduced to

$$
\begin{aligned}
& -t \sum_{m, \delta= \pm 1} g\left(\left\{x_{1}, \ldots, x_{m}+\delta, \ldots, x_{N_{e}}\right\},\left\{\gamma_{m}\right\}\right) \\
& \quad+U_{\text {eff }} \sum_{n<m} \delta_{x_{n}, x_{m}}\left(1-\delta_{\gamma_{n}, \gamma_{m}}\right) g\left(\left\{x_{m}\right\},\left\{\gamma_{m}\right\}\right) \\
& =\left(E_{g}-N_{e} J / 2-N_{\wedge} s\right) g\left(\left\{x_{m}\right\},\left\{\gamma_{m}\right\}\right),
\end{aligned}
$$

where $E_{g}$ is the ground-state energy of the model. In the case of ferromagnetism, the Schrödinger equation is reduced to a one-band Hubbard model with the on-site Coulomb interaction, $U_{\text {eff }}=U^{\prime}-J$ if we use the orbit indices instead of usual spin indices. This equation can be solved exactly by means of the Bethe ansatz. ${ }^{12}$ The solution to $g$ is expressed as

$$
g\left(\left\{x_{m}\right\},\left\{\gamma_{m}\right\}\right)=\sum_{P}[Q, P] \exp \left[i \sum_{m=1}^{N_{e}} k_{P_{m}} x_{Q_{m}}\right],
$$

where $P$ and $Q$ are two permutations of $\left(1,2, \ldots, N_{e}\right)$. The coefficients $[Q, P]$ have the relation

$$
\begin{gathered}
{[Q, P]=Y_{n m}^{i, i+1}\left[Q, P^{\prime}\right] ;} \\
Y_{n m}^{i, i+1}=\frac{\left(\sin k_{n}-\sin k_{m}\right) P^{i, i+1}-i U_{\mathrm{eff}} / 2}{\left(\sin k_{n}-\sin k_{m}\right)+i U_{\mathrm{eff}} / 2},
\end{gathered}
$$

where

$$
\begin{aligned}
P & =\left(P_{1}, \ldots P_{i}=n, P_{i+1}=m, \ldots P_{N}\right) \\
P^{\prime} & =\left(P_{1}, \ldots P_{i}^{\prime}=m, P_{i+1}^{\prime}=n, \ldots P_{N}\right) .
\end{aligned}
$$

$k_{n}\left(n=1, \ldots, N_{\wedge}\right)$ are determined by Eqs. (11) and (12). The ground state is located in the subspace $N_{1}=N_{2}$ if the number of electrons is even, or $N_{1}=N_{2} \pm 1$ if the number of electrons is odd. Its lowest energy is

$$
E_{g}=-2 t \sum_{n=1}^{N_{e}} \cos k_{n}-\frac{1}{2} N_{e} J-N_{\wedge} J_{H} S .
$$

The properties of the Lieb-Wu's solution have been discussed extensively. ${ }^{13}$ Except for the filling $N_{e}=N_{\wedge}$, there is no energy gap. Hence the ground state is a metallic ferromagnet.

We have obtained the exact solution of the ground state of the electronic model for transition-metal oxides in a onedimensional open chain for any density of electrons in the case of $U=+\infty$. The state is proven to be fully ferromagnetic. Here we come to discuss the physical mechanism of ferromagnetism and its possible relevance to realistic and three-dimensional systems. The model we discuss contains all possible factors which favor ferromagnetism: large onsite Coulomb interaction, orbital degeneracy of itinerant electrons and the Hund's rule coupling. In the case of $J$ $=J_{H}=0$, the model has a highly spin-degenerated ground state when $U=+\infty$. Any finite $U$ will remove the degeneracy to form a spin singlet state. Thus without the Hund's rule coupling the only strong on-site Coulomb interaction cannot drive electrons to form ferromagnetism. On the contrary, the nonzero Hund's rule coupling, i.e., $J \neq 0$ and $J_{H}$ $\neq 0$, will also remove the degeneracy to form a ferromagnet. This indicates that the interplay between the on-site Coulomb interaction and the Hund's rule coupling plays an essential role in the stability of ferromagnetism in the model.

The ferromagnetism may also be survived in the case of finite $U$ and large $J$ and $J_{H}$. To see this effect, we consider a more realistic and three-dimensional model which is used extensively to describe the doped lanthanum manganese oxides. The final consequences are also valid to the onedimensional case. The transfer integrals in the model for $R_{1-x} X_{x} \mathrm{MnO} 3$ are assumed to take a Slater-Koster form given by the hybridization between $e_{g}$ orbit and nearest oxygen $p$ orbit $^{14}$

$$
t_{i j}^{\gamma \gamma^{\prime}}=-t \tau_{\gamma \gamma^{\prime}}^{x},-t \tau_{\gamma \gamma^{\prime}}^{y},-t \tau_{\gamma \gamma^{\prime}}^{z}
$$

for $\mathbf{r}_{j}=\mathbf{r}_{i} \pm \hat{x}, \mathbf{r}_{i} \pm \hat{y}$ and $\mathbf{r}_{i} \pm \hat{z}$, respectively, where

$\tau^{x}=\left(\begin{array}{cc}\frac{1}{4} & -\frac{\sqrt{3}}{4} \\ -\frac{\sqrt{3}}{4} & \frac{3}{4}\end{array}\right), \quad \tau^{y}=\left(\begin{array}{cc}\frac{1}{4} & \frac{\sqrt{3}}{4} \\ \frac{\sqrt{3}}{4} & \frac{3}{4}\end{array}\right), \quad \tau^{z}=\left(\begin{array}{cc}1 & 0 \\ 0 & 0\end{array}\right)$.

$\gamma= \pm 1$ represent $\left(\begin{array}{l}1 \\ 0\end{array}\right)=3 z^{2}-r^{2}$ orbit and $\left(\begin{array}{l}0 \\ 1\end{array}\right)=x^{2}-y^{2}$ orbit of the $e_{g}$ electron, respectively. In reality, the parameters of the model for the manganites are roughly estimated as $U$ $\gg J_{H}, J \gg t$. Hence we can obtain an effective Hamiltonian at $N_{e}=N_{\wedge}$ by means of combination of the projection technique and the perturbation technique ${ }^{15}$

$$
\begin{aligned}
H_{\mathrm{eff}}= & -\frac{t^{2}}{U^{\prime}-J / 2} \sum_{i j}\left(\frac{\mathcal{S}_{i} \cdot \mathcal{S}_{j}+(S+1 / 2)(S+3 / 2)}{(S+1 / 2)(S+3 / 2)}\right) P_{i j}^{d} \\
& +\frac{t^{2}}{U^{\prime}+3 J / 2+J_{H} S} \sum_{i j}\left(\frac{\mathcal{S}_{i} \cdot \mathcal{S}_{j}-(S+1 / 2)^{2}}{(S+1 / 2)(S+3 / 2)}\right) P_{i j}^{d} \\
& +\frac{t^{2}}{U+J_{H} S} \sum_{i j}\left(\frac{\mathcal{S}_{i} \cdot \mathcal{S}_{j}-(S+1 / 2)^{2}}{(S+1 / 2)^{2}}\right) P_{i j}^{s}
\end{aligned}
$$

where $\mathcal{S}_{i}$ is a spin operator with $S+1 / 2$ as a ferromagnetic combination of the localized spin and itinerant electron at the same site. $P_{i j}^{s(d)}$ is the projection operator for the orbital occupancy

$$
\begin{gathered}
P_{i j}^{s}=\tau_{i}^{\alpha} \tau_{j}^{\alpha} ; \\
P_{i j}^{d}=\tau_{i}^{\alpha}\left(1-\tau_{j}^{\alpha}\right)
\end{gathered}
$$

with $\alpha=x, y, z$, which depends on the direction of $\mathbf{r}_{i}-\mathbf{r}_{j}$. From the effective Hamiltonian, the first term favors ferro- 
magnetic correlation and the other two terms favor antiferromagnetic correlation. When $U=+\infty$ or sufficiently large, the third term is suppressed. Comparing the first and second terms, we find that the ferromagnetic coupling is always stronger than antiferromagnetic coupling since

$$
\frac{t^{2}}{U^{\prime}-J / 2}>\frac{t^{2}}{U^{\prime}+3 J / 2+J_{H} S}
$$

and $P_{i j}^{d}$ always has nonnegative eigenvalues. When $J_{H} S$ is sufficiently large and $U^{\prime}-J / 4$ is kept unchanged, the first term is predominant even for a finite $U$. However, when $U$ decreases to finite, an antiferromagnetic frustration is introduced. The stability of ferromagnetism depends on the competition between the strong Hund coupling and the effect of finite $U$. In $\mathrm{LaMnO}_{3}$, where $N_{e}=N_{\wedge}$, a ferromagnetic and insulating phase was observed at low temperatures. ${ }^{16}$ This experimental observation is in qualitative agreement with our analysis. Oppositely, if we neglect the orbital degeneracy of $e_{g}$ electrons and use a one-band Kondo lattice model to describe the sample, we always have an antiferromagnetic phase at low temperatures. ${ }^{17}$ It implies that the orbital degree of freedom is very important for us to understand the phase diagrams of doped manganese oxides. When the system is doped, i.e. $N_{e}<N_{\wedge}$, due to the strong Hund coupling, $J_{H}$ $\gg t$, the motion of itinerant electrons tends to force the localized spins to align parallell. Thus the double-exchange mechanism as well as the orbital degeneracy and strong $U$ should be also responsible for the metallic ferromagnetism in $R_{1-x} X_{x} \mathrm{MnO}_{3}$. The detailed discussion of the phase diagrams will be published elsewhere.

Summarizing, the model on an open chain is solvable in the case of $U=+\infty$. The ground state is a metallic ferromagnetic except for the density $N_{e} / N_{\wedge}=1$. The charge and orbital degrees of freedom are determined by a Lieb-Wu solution for the one-dimensional Hubbard model. The ferromagnetism can be survived in finite $U$ and higher dimensional systems. Due to the orbital degeneracy of itinerant electrons and strong on-site repulsion $U$, the superexchange coupling can be ferromagnetic rather than antiferromagnetic in the one-band case.

This work was supported by a CRCG research grant at the University of Hong Kong.
${ }^{1}$ M. C. Gutzwiller, Phys. Rev. Lett. 10, 59 (1963); J. Hubbard, Proc. R. Soc. London, Ser. A 276, 238 (1963); J. Kanamori, Prog. Theor. Phys. 30, 275 (1963).

${ }^{2}$ J. C. Slater, Phys. Rev. 49, 537 (1936); J. H. van Vleck, Rev. Mod. Phys. 25, 220 (1953); L. M. Roth, Phys. Rev. 149, 306 (1966).

${ }^{3}$ C. Zener, Phys. Rev. 82, 403 (1951); P. W. Anderson and H. Hasegawa, ibid. 100, 675 (1955); K. Kubo and N. Ohata, J. Phys. Soc. Jpn. 33, 21 (1972).

${ }^{4}$ H. Tasaki, Prog. Theor. Phys. 99, 489 (1998).

${ }^{5}$ D. Vollhardt, N. Bümer, K. Held, M. Kollar, J. Schlipf, and M. Ulmke, Z. Phys. B 103, 283 (1997).

${ }^{6}$ S. Q. Shen, Int. J. Mod. Phys. B 12, 709 (1998).

${ }^{7}$ K. I. Kugel and D. I. Khomskii, Sov. Phys. JETP 37, 725 (1973); S. Ishihara, J. Inoue, and S. Maekawa, Physica C 263, 130 (1996); Phys. Rev. B 55, 8280 (1997); R. Shiina, T. Nishitani, and H. Shiba, J. Phys. Soc. Jpn. 66, 3159 (1997); S. Ishihara, M.
Yamanaka, and N. Nagaosa, Phys. Rev. B 56, 686 (1997).F. Zhong, J. Dong, and Z. D. Wang, ibid. 58, 15310 (1998).

${ }^{8}$ See B. Simon, Statistical Mechanics of Lattice Gas (Princeton University Press, Princeton, New Jersey, 1993).

${ }^{9}$ K. Kubo, J. Phys. Soc. Jpn. 51, 782 (1982).

${ }^{10}$ H. Tasaki, Phys. Rev. B 40, 9192 (1989).

${ }^{11}$ S. Q. Shen, Phys. Rev. B 57, 6474 (1998).

${ }^{12}$ E. H. Lieb and F. Y. Wu, Phys. Rev. Lett. 20, 1445 (1968).

${ }^{13}$ See V. E. Korepin and F. H. L. Essler, Exactly Solvable Models of Strongly Correlated Electrons (World Scientific, Singapore, 1994).

${ }^{14}$ H. Shiba, R. Shiina, and A. Takahashi, J. Phys. Soc. Jpn. 66, 941 (1997).

${ }^{15}$ S. Q. Shen and Z. D. Wang, Phys. Rev. B 58, R8877 (1998).

${ }^{16}$ J. B. Goodenough, Phys. Rev. 100, 564 (1955); P. Schiffer et al., Phys. Rev. Lett. 75, 3336 (1995).

${ }^{17}$ S. Q. Shen, Phys. Rev. B 53, 14252 (1996); 55, 14330 (1997). 